\title{
Natural course of excessive genu varum without underlying pathology in children
}

\author{
Si-Wook Lee ${ }^{1 *}$, Kwang-Soon Song ${ }^{2}$, Kyung-Jae Lee ${ }^{3}$, Chang-Jin Yon ${ }^{4}$ and Hyuk-Jun Kwon ${ }^{5}$ \\ ${ }^{1} \mathrm{Si}-W o o k$ Lee, MD, PhD; Keimyung University, Dongsan Hospital, Orthopedic Surgery, Daegu, Dalseo-gu, Korea \\ ${ }^{2}$ Kwang-Soon Song, MD, PhD; Keimyung University, Dongsan Hospital, Orthopedic Surgery, Daegu, Dalseo-gu, Korea \\ ${ }^{3}$ Kyung-Jae Lee, MD, PhD; Keimyung University, Dongsan Hospital, Orthopedic Surgery, Daegu, Dalseo-gu, Korea. \\ ${ }^{4}$ Chang-Jin Yon, MD; Keimyung University, Dongsan Hospital, Orthopedic Surgery, Daegu, Dalseo-gu, Korea \\ ${ }^{5}$ Hyuk-Jun Kwon, MD; Keimyung University, Dongsan Hospital, Orthopedic Surgery, Daegu, Dalseo-gu, Korea
}

\begin{abstract}
Background: Despite genu varum's status as a common pediatric problem, there remain no serial studies investigating the natural course of excessive physiologic genu varum in children. This study, therefore, aims to identify the natural course of the anatomical tibiofemoral angle (aTFA) in children with excessive physiologic genu varum without any underlying pathological conditions.

Materials and methods: Between 1997 and 2008, among 70 children who had been referred from a primary hospital to evaluate angular deformity of the knee, 29 consecutive limbs (10 bilateral and 9 unilateral) of 19 healthy Korean children with excessive physiologic genu varum equal to or greater than 2 standard deviations (SDs) of the aTFA at their initial visit until all children were 6 years of age. We analyzed the value of the aTFA as well as the changes in patterns, and compared our findings with results from previous studies.

Results: The mean aTFA at the initial visit was $18^{\circ}$ varus (range: $9^{\circ}$ to $36^{\circ}$ ), the mean age of normalization was 3.2 years, and the mean value of the aTFA was varus $17.2^{\circ}$ at the age of 1 year, followed by a steady decrease to $0^{\circ}$ at almost the age of 3.3 years. Starting at the age of 4 years, the aTFA was a mean of $5.2^{\circ}$ genu valgum and gradually changed until the age of 6 years, where it was sustained at an average of $3.4^{\circ}$ genu valgum.
\end{abstract}

Conclusion: Excessive physiological genu varum exceeding 2 SD in young Korean children improved spontaneously without the need for prosthetics or orthotics.

\section{Introduction}

Genu varum is a common pediatric problem that results in consultations regarding the need for surgery. Although in the majority of cases, genu varum is physiologic and resolves spontaneously, physicians need to differentiate between physiologic angular variations and pathological conditions that require special evaluation and treatment [1-6]. Although physiological changes in knee alignment are well established, inexperienced physicians cannot be confident of spontaneous recovery and may recommend ineffective and harmful intervention, such as bracing, in cases of excessive physiologic genu varum [6-8]. Several studies have been conducted to investigate mean values and overall physiologic changes in the anatomical tibiofemoral angle (aTFA) by age [9-13]. To our knowledge, however, there have been no serial studies investigating the natural course of excessive physiologic genu varum in children. Therefore, we conducted this study to identify the mean values and natural course of the aTFA in excessive physiologic genu varum exceeding more than 1 standard deviation (SD) from the normal value for the aTFA in Korean children [12]. Afterward, we compared our findings with recently reported generalized overall changes in the aTFA in Korean children as well as findings of studies in other countries [2,6,8-16].

\section{Materials and methods}

A retrospective study approved the deliberation of the ethics committee (IRB No. 2013-07-058) from September 6, 2013, to September
5, 2014. Between 1997 and 2008, among 70 children who were referred to our hospital for excessive genu varum at the recommendation of a primary physician or the request of a concerned parent, we reviewed 29 consecutive limbs (10 bilateral and 9 unilateral) of 19 healthy Korean children (11 boys and 8 girls) with excessive physiologic genu varum equal to or greater than 2 SDs of the aTFA at their initial clinical visit and were monitored until all children were 6 years of age, procuring all necessary follow-up data for the children throughout this period. We defined excessive physiologic genu varum as the presence of genu varum of more than 2 SDs and without any underlying pathological conditions. Patients whose genu varum was associated with underlying pathological conditions and who had experienced bracing or the use of an insole were excluded.

The children were grouped according to age, and their chronological age was rounded off to the nearest integer, similar to the method of a previous study regarding the development of normal aTFA in Korean children [12]. In this study, those whose ages ranged from 2 years and

${ }^{\star}$ Correspondence to: $\mathrm{Si}-$ Wook Lee, $\mathrm{MD}, \mathrm{PhD}, 1035$ Dalgubeol-daero, Keimyung University, Dongsan Hospital, Orthopedic Surgery, Daegu, Dalseo-gu, Korea, E-mail: shuk2000@naver.com

Key words: excessive physiologic genu varum, children, spontaneous resolution, $a T F A$

Received: June 03, 2020; Accepted: June 29, 2020; Published: July 03, 2020 
7 months to 3 years and 6 months were placed in the group of 3-yearolds.

In general, full-length standing teleroentgenograms were obtained with the patient standing and the patella pointing straight ahead, indicating the neutral position of the lower extremity. However, for participants who were too young to stand, radiographs were obtained in the supine position. The degree of varus was defined as the bisecting angle between the intramedullary lines of the femur and the tibia. The serial tibiofemoral angle (TFA) was measured (146 measurements) with the use of a Picture Archiving and Communication System (PACS) network (Marosis, DICOH version 3.0; INFINITT, Seoul, Korea) and the average was used for subsequent analysis. Serial aTFAs were measured and compared with the results from previous studies [8]. All measurements were made by two examiners using standard techniques to minimize inter- and intra-examiner bias. The neutralization time of the aTFA and the difference between neutralizing times of the aTFA by gender were analyzed. The changing pattern of the aTFA was assessed and compared with findings from previous studies.

Statistical analysis was performed using SPSS software package 15.0 (IBM, Armonk, NY, USA). To examine patients' baseline characteristics, we analyzed the aTFA and descriptive statistics. To determine the correlation between the TFA and various parameters such as age, sex, duration until normalization, and various values of the aTFA in different ethnic groups, we used the independent $t$-test and one-way analysis of variance. The mean, SD, and $95 \%$ confidence intervals were calculated for age and sex. Statistical significance was set at a value of $p<0.05$. All statistical analysis was performed by a single statistician.

\section{Results}

The mean age of participants at the initial office visit was 17.7 months (range: 9 to 36 months), and the mean duration of follow-up was 40.9 months (range: 10 to 70 months). The mean aTFA at the initial visit was $18^{\circ}$ (range, $9^{\circ}$ to $36^{\circ}$ ), and the mean time elapsed from the initial visit to normalization was 20.3 months. The mean age of normalization was 3.2 years. Regarding changes in aTFA according to age, the mean aTFA was $17.2^{\circ}$ varus at 1 year, followed by a steady decrease to an aTFA of $0^{\circ}$ at almost 3.3 years. At the age of 4 years, the aTFA was $5.2^{\circ}$ of valgus and gradually changed until the age of 6 years, where it remained at an average of $3.4^{\circ}$ genu valgum (Figure 1).

The overall pattern of the chronological changes in the aTFA was similar to that found in previous studies; however, the physiological genu varum was converted to genu valgum at between 3 and 4 years of age. Neutralization of the aTFA $\left(\right.$ TFA $=0^{\circ}$ ) was seen at an average of 3.3 years of age. Moreover, there was no period of the peak valgus at age 3 to 4 years in a normative pattern. The final average TFA was $3.4^{\circ}$ valgus after six years of follow-up. All limbs spontaneously recovered to the normal range for the aTFA when the participants were 6 years of age (Figure 2).

\section{Discussion and conclusions}

Genu varum is a common pediatric concern prompting referrals to specialists to address parents' doubts. Whether the varus positioning is caused by a transient physiologic condition or by pathological conditions such as infantile tibia vara (Blount disease), rickets, or focal fibrocartilaginous dysplasia, accurate diagnosis is critical because treatment and prognosis differ per etiology [17].

Age-related overall patterns in TFA change, along with mean values and normal ranges of TFAs in growing children of different ethnic

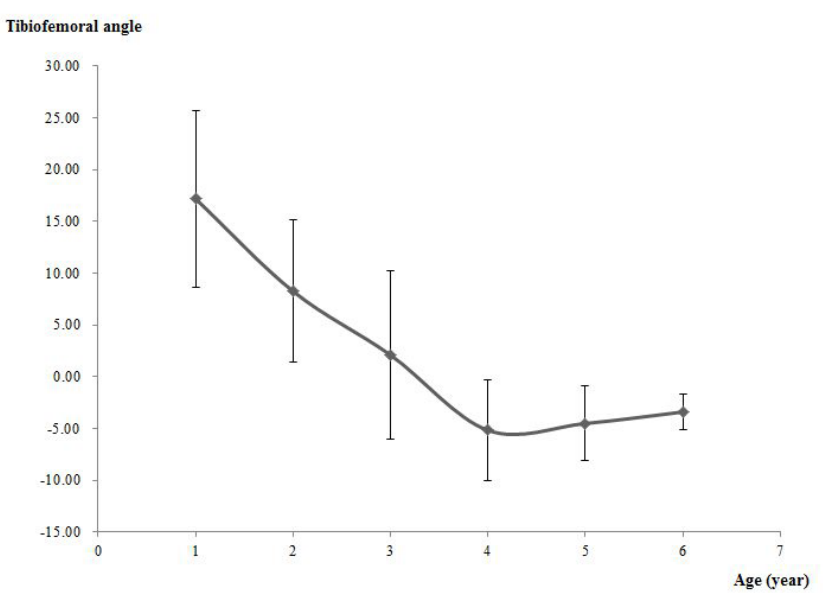

Figure 1. The mean value and standard deviations of anatomical tibiofemoral angles by age in excessive genu varum without underlying pathology. Regarding changes in the anatomical tibiofemoral angle (aTFA) according to age, the mean aTFA was $17.2^{\circ}$ varus at 1 year, followed by a steady decrease to an aTFA of $0^{\circ}$ at almost 3.3 years. At the age of 4 years, the aTFA was $5.2^{\circ}$ of valgus and gradually changed until the age of 6 years, where it remained at an average of $3.4^{\circ}$ genu valgum

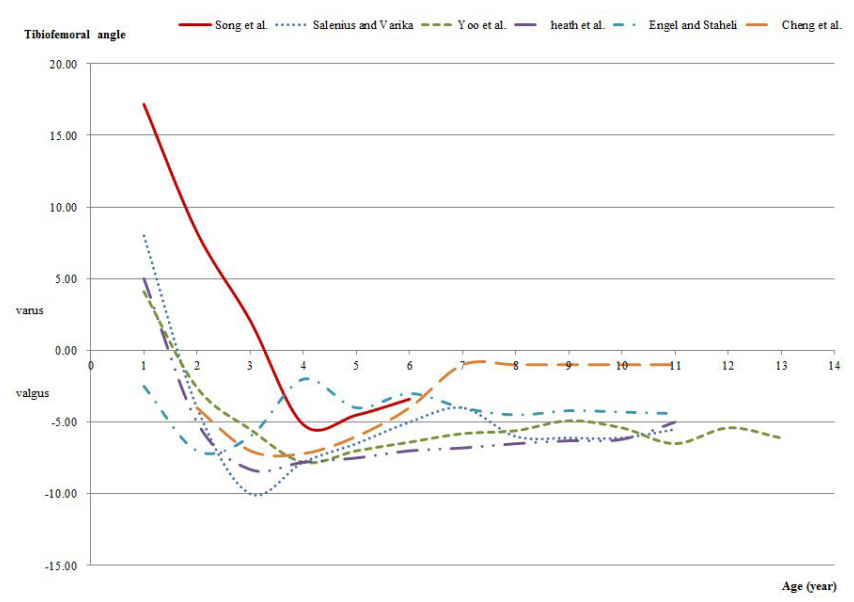

Figure 2. The overall pattern of the chronological changes in the anatomical tibiofemoral angle was similar to that found in previous studies; however, the physiological genu varum was converted to genu valgum at between 3 and 4 years of age

groups, have already established by several researchers $[6,9,10,12$ 14,16]. Overall chronological changes and spontaneous resolution of physiologic genu varum have been documented by many excellent studies $[7-9,18,19]$. However, it is not easy for inexperienced orthopedic surgeons to be confident that excessive physiologic genu varum will spontaneously resolve. Thus, we investigated the natural course of excessive genu varum to confirm whether it always spontaneously resolves.

In the evaluation of knee alignment, less invasive and less expensive clinical methods have been used, including measurement of the TFA and the intermalleolar and intercondylar distance $[6,9,14-16,20]$. However, we used radiological analysis for children with excessive physiologic genu varum because it was necessary to obtain more accurate radiological references $[10-13,18]$, to rule out underlying pathological conditions, to investigate serial changes in the aTFA objectively, and to compare results with the normative data on chronological changes in knee angles that have already been studied using radiographs. 
The results from previous studies show a different pattern of change in TFA according to ethnicity $[6,8,9,11-14,16,18,20]$. The overall pattern in chronological changes in the knee angle that we found were similar to those described previously in Korean [12] and Western $[9,11]$ populations, but it differed from those described in Turkish, Nigerian, and Indian $[6,14,15]$ populations.

The time to the neutralization of the TFA in our study was an average of 1.4 years longer than the Korean average [12] and was similar to the Japanese average [13]. The physiologic genu varum period in our study was also prolonged compared with normative data on chronological changes in knee angles in Korea. Characteristically, there was no normative peak valgus period at ages 3 to 4 years [11,12,19], owing to the delayed and steady decline of the TFA between the ages of 3 and 6 years (Figure 3). Although we found that time for the neutralization of the aTFA and duration of normalization differed between boys and girls, the difference was not statistically significant.

An excessive physiologic genu varum exceeding 2 SDs in young children improved spontaneously without any treatment. Despite prolongation of the genu varum period, a lack of a definite physiologic genu valgum period, and the delayed neutralization of the aTFA by 1.5 years, the aTFA in our population became similar to that previously reported for other populations: $6^{\circ}$ valgus at age 6 years. The natural course and spontaneous resolution of excessive genu varum in our study may serve as a guideline for physicians treating excessive genu varum in young children. Because excessive physiological genu varum exceeding 2 SDs in young Korean children improved spontaneously without the need for prosthetics or orthotics, there is no need for the use of artificial limbs in this case.

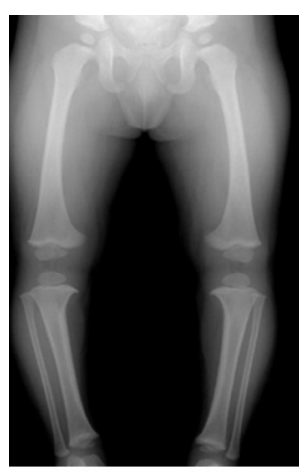

18 months

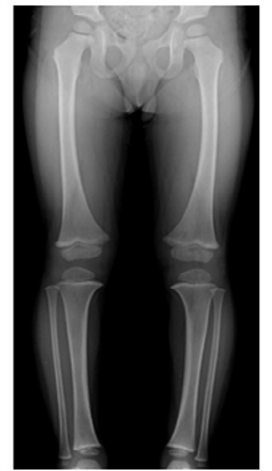

35 months

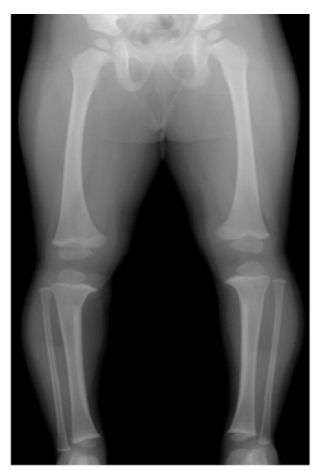

24 months

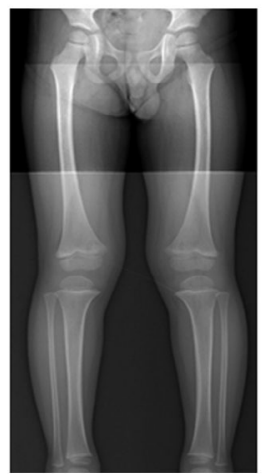

47 months

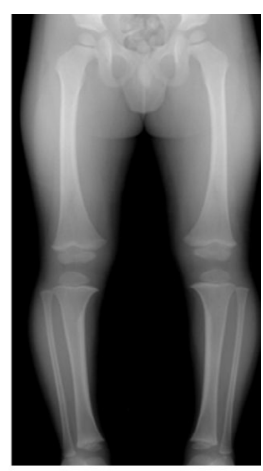

30 months

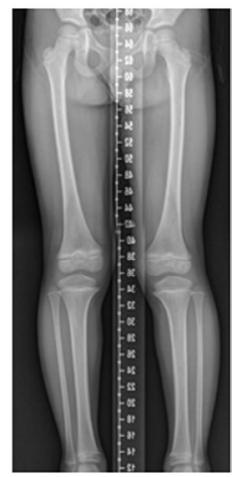

83 months
Figure 3. An 18-month-old boy had severe genu varum without definite pathology. Over time, the varus deformity decreased with aging and without treatment, neutralizing by 35 months and normalizing by 85 months. There was no definite genu valgum period

\section{Conflicts of interest}

The authors declare that they have no conflict of interest.

\section{Acknowledgment}

None.

\section{References}

1. Brooks WC, Gross RH (1995) Genu varum in children: Diagnosis and treatment. $J$ Am Acad Orthop Surg 3(6): 326-335. [Crossref]

2. Do TT (2001) Clinical and radiographic evaluation of bowlegs. Curr Opin Pediatr 13 42-46. [Crossref]

3. Greene WB (1994) Genu varum and genu valgum in children. Instr Course Lect 43 $151-159$

4. Kling TF Jr, Hensinger RN (1983) Angular and torsional deformities of the lower limbs in children. Clin Orthop Relat Res: 136-147. [Crossref]

5. Fabry G (2010) Clinical practice. Static, axial, and rotational deformities of the lower extremities in children. Eur J Pediatr 169: 529-534.

6. Saini UC, Bali K, Sheth B, Gahlot N, Gahlot A (2010) Normal development of the knee angle in healthy Indian children: a clinical study of 215 children. $J$ Child Orthop 4: 579-586. [Crossref]

7. McDade W (1977) Bow legs and knock knees. Pediatr Clin North Am 24: 825-839.

8. Morley AJ (1957) Knock-knee in children. Br Med J 2: 976-979.

9. Heath CH, Staheli LT (1993) Normal limits of knee angle in white children--genu varum and genu valgum. J Pediatr Orthop 13: 259-262

10. Salenius P, Vankka E (1975) The development of the tibiofemoral angle in children. $J$ Bone Joint Surg Am 57: 259-261. [Crossref]

11. Vankka E, Salenius P (1982) Spontaneous correction of severe tibiofemoral deformity in growing children. Acta Orthop Scand 53: 567-570. [Crossref]

12. Yoo JH, Choi IH, Cho TJ, Chung CY, Yoo WJ (2008) Development of tibiofemoral angle in Korean children. J Korean Med Sci 23: 714-717.

13. Hachiya M (1981) A roentgenographical study on chronological changes in genu varum and valgum in children (author's transl). Nihon Seikeigeka Gakkai Zasshi 55: 31-43. [Crossref]

14. Arazi M, Ogun TC, Memik R (2001) Normal development of the tibiofemoral angle in children: a clinical study of 590 normal subjects from 3 to 17 years of age. J Pediatr Orthop 21: 264-267. [Crossref]

15. Omololu B, Tella A, Ogunlade SO, Adeyemo AA, Adebisi A, et al. (2003) Normal values of knee angle, intercondylar and intermalleolar distances in Nigerian children. West Afr J Med 22: 301-304.

16. Cheng JC, Chan PS, Chiang SC, Hui PW (1991) Angular and rotational profile of the lower limb in 2,630 Chinese children. J Pediatr Orthop 11: 154-161.

17. Sherman M (1960) Physiologic bowing of the legs. South Med J 53: 830-836.

18. Herring JA, Tachdjian MO, Children. TSRHf (2008) Tachdjian's pediatric orthopaedics. 4th edn. Saunders/Elsevier, Philadelphia.

19. Engel GM, Staheli LT (1974) The natural history of torsion and other factors influencing gait in childhood. A study of the angle of gait, tibial torsion, knee angle, hip rotation, and development of the arch in normal children. Clin Orthop Relat Res: 12-17. [Crossref]

20. Cahuzac JP, Vardon D, Sales de Gauzy J (1995) Development of the clinical tibiofemoral angle in normal adolescents. A study of 427 normal subjects from 10 to 16 years of age. J Bone Joint Surg Br 77: 729-732. [Crossref]

Copyright: (C2020 Lee S. This is an open-access article distributed under the terms of the Creative Commons Attribution License, which permits unrestricted use, distribution, and reproduction in any medium, provided the original author and source are credited. 